\title{
An expeditious synthesis of methyl 5-(2-amino 4-arylthiazolyl) acetates using microwave irradiation
}

\author{
Mahesh Attimarad ${ }^{a *}$ and S. Mohan ${ }^{b}$ \\ ${ }^{a} \mathrm{Al}$-Ameen College of Pharmacy, Hosur Road, Bangalore-560 027, India \\ E-mail : Mattimarad@yahoo.com \\ ${ }^{b}$ PES College of Pharmacy, Hanumanth Nagar, Bangalore-560 050, India \\ Manuscript received 4 July 2005, revised 19 September 2006, accepted 21 September 2006
}

\begin{abstract}
Trisubstituted thiazole derivatives can be prepared conveniently by condensation of methyl 3-bromo3-aroyl propionates with thiourea and (un)substituted phenyl thiourea under microwave irradiation in good yields. The structures of the newly synthesized compounds are characterized by spectral data and elemental analysis.
\end{abstract}

Keywords : Microwave irradiation, thiazole, thiourea.

Thiazole derivatives are reported to show a broad spectrum of biological activity, which includes antimicrobial ${ }^{1}$, antidiabetic ${ }^{2}$, analgesic and anti-inflammatory ${ }^{3}$ activities. The introduction of thiazole ring improved the activity as in meloxicam in the oxicam series of anti-inflammatory drugs $^{4}$. Thiazoles are easily metabolized by routine biochemical reactions and are noncarcinogenic in nature ${ }^{5}$. In view of their safety and importance, there is a continuing interest in developing versatile synthetic routes. Generally, 2-aminothiazoles are synthesized by the condensation of a halo carbonyl compound with thiourea, known as Hantzsch synthesis ${ }^{6}$ and has a wide scope, permitting the synthesis of a variety of thiazole derivatives.

In this paper, we wish to report a novel synthesis of methyl 5-(2-amino 4-arylthiazolyl) acetates (4a-r) from methyl 3-bromo-3-aroylpropionates (2) and (un)substituted phenyl thioureas (3a-i) (Scheme 1) by MORE chemistry ${ }^{9}$ using PEG-400 as a solvent. PEG-400 is miscible with water thereby simplifying the workup. Furthermore it is inexpensive and readily available in bulk quantities.

\section{Results and discussion}

The methyl 3-aroylpropionates 1c-d were prepared in better yields by irradiating a mixture of 3-aroylpropionic acids 1a-b, concentrated sulphuric acid and methanol in open glass containers using unmodified household microwave oven in $1.5 \mathrm{~min}$. This procedure considerably reduces the longer reaction time ( 7 hours of refluxing ${ }^{10}$ ) usually encountered in traditional ester synthesis. The acid is then brominated to methyl 3-bromo-3- aroylpropionate $\mathbf{2 a - b}$. The reaction of 3-bromo-3aroylpropionate with (un)substituted phenyl thiourea 3a$i$ in methanol at reflux resulted in the formation of thiazoles acetates $4 a-i$ and $4 j-r$ with $65-78 \%$ yield in $60-75$ $\mathrm{min}$. However, when the reaction was performed in PEG400 in microwave oven the reaction time was reduced to 0.5-1.5 min and the yield rose to $85-95 \%$. The reaction mixture was exposed to the microwave irradiation in an open vessel covered with stemless glass funnel at lower power $(540 \mathrm{~W})$. The long neck of glass vessel remains cool since glass is transparent to microwaves. A beaker of water was kept near the reaction vessel to serve as "heat sink" to provide fine control of the temperature of the reaction mixture ${ }^{11}$.

We have also attempted the above reaction in PEG200 , ethylene glycol, diethylene glycol, DMSO, isopropyl alcohol and without any solvent (Table 1). The maximum yield of the desired product was obtained when the reaction was performed in PEG-400.

To test the generality of the reaction a variety of phenyl thioureas (3a-i) were condensed with bromo propionates in PEG-400 under microwave irradiation to form the corresponding methyl 4-aroyl-2-arylamino thiazolyl acetates (Table 2). When two electron withdrawing groups were placed in phenyl thiourea 3 the yields of substituted thiazolyl acetates $\mathbf{4 g}-\mathrm{h}$ and $\mathbf{4 p - q}$ were slightly less and the rate of reaction was also slower. 
Table 1. Reaction of methyl 3-bromo-3-(4-chlorobenzoyl) propionate- with 2-chlorophenyl thiourea in various solvents under microwave irradiation for $1 \mathrm{~min}$ at $540 \mathrm{~W}$

$\begin{array}{llc}\text { Entry } & \text { Solvent } & \text { Yield \% (4b) } \\ \text { 1. } & \text { PEG-400 } & 92 \\ \text { 2. } & \text { PEG-200 } & 82 \\ 3 . & \text { Ethylene glycol } & 71 \\ \text { 4. } & \text { Diethylene glycol }^{a} & 65 \\ \text { 5) } & \text { Isopropyl alcohol }^{a} & 80 \\ \text { 7. } & \text { DMSO } & 74 \\ & \text { Without solvent } & 45\end{array}$

${ }^{a}$ Carefully irradiated in long neck open vessel covered with stemless glass funnel to avoid the evaporation of IPA.

\section{Experimental}

Melting points are uncorrected and were recorded in liquid paraffin bath using open end capillaries. Thin layer chromatography was performed on silica gel G. A simple household microwave (BPL Sanyo India) oven (900 W) equipped with a turntable was used for irradiation. The IR spectra were run on Shimadzu FT IR spectrophotometer in $\mathrm{KBr}$ pellets. ${ }^{1} \mathrm{H}$ NMR spectra were obtained using Jeol GSX-400 FT NMR $400 \mathrm{MHz}$ in $\mathrm{CDCl}_{3} / \mathrm{DMSO}-$ $d_{6}$ solvent using TMS as internal reference. Mass spectra were recorded by Jeol-JMS-300 spectrometer at $70 \mathrm{eV}$.
Methyl 3-(4-chlorobenzoyl)propionate (1c) : 3-(4Chlorobenzoyl)propionic acid 1a $(2.1 \mathrm{~g}, 10 \mathrm{mmol})$, dry methanol $(20 \mathrm{ml})$ and concentrated sulphuric acid $(0.5$ $\mathrm{ml}$ ) were taken in a long neck conical flask covered with stemless funnel to avoid excessive evaporation. The resulting mixture was irradiated in a domestic microwave oven at $200 \mathrm{~W}$ for $1.5 \mathrm{~min}$. After that the reaction mixture was cooled to room temperature and poured into ice cold water. The ester 1c was extracted into ether and washed with water, sodium bicarbonate solution and the ether layer was separated, dried and distilled off to get methyl 3-(4-chlorobenzoyl)propionate 1c (m.p. $62-63^{\circ} \mathrm{C}$, $96 \%$, lit. ${ }^{12}$ m.p. $\left.63{ }^{\circ} \mathrm{C}, 75 \%\right)$.

Methyl 3-bromo-3-(4-chlorobenzoyl)propionate (2a) : Methyl 3-(4-chlorobenzoyl)propionate $1 \mathrm{c}$ ( $2.2 \mathrm{~g}, 10 \mathrm{mmol})$ was broninated in warm chloroform $(20 \mathrm{ml})$ by addition of bromine $(1.7 \mathrm{~g}, 11 \mathrm{mmol})$ dropwise with stirring over $30 \mathrm{~min}$, stirring was continued for another $2 \mathrm{~h}$. The reaction mixture was washed with water and then chloroform layer was dried over sodium sulpahte and distilled to get methyl 3-bromo-3-(4-chlorobenzoyl)propionate $\mathbf{2 a}$ as viscous oil which was used as such for the next step. Yield : 95\% (Scheme 1).

Methyl 2-phenylamino-4-(4-chlorophenyl)thiazole-5acetate (4a) : A slurry of bromo propionate $2 \mathrm{a}(900 \mathrm{mg}$, $3 \mathrm{mmol}$ ) and phenyl thiourea $3 \mathrm{a}(450 \mathrm{mg}, 3 \mathrm{mmol})$ in PEG-400 $(10 \mathrm{ml})$ was irradiated at $540 \mathrm{~W}$ for 1 min.

\begin{tabular}{|c|c|c|c|c|c|c|}
\hline & & & Physica & pounds & & \\
\hline Compd. & & & Time & M.p. & & \\
\hline & $\mathrm{x}$ & $Y$ & $(\min )$ & $\left({ }^{\circ} \mathrm{C}\right)$ & MW & Conventional \\
\hline $4 a$ & $\mathrm{Cl}$ & $\mathrm{H}$ & 1.0 & $119-120$ & 93 & 69 \\
\hline $4 b$ & $\mathrm{Cl}$ & $2-\mathrm{Cl}$ & 0.5 & $97-98$ & 92 & 73 \\
\hline $4 c$ & $\mathrm{Cl}$ & $4-\mathrm{Br}$ & 1.0 & $147-148$ & 89 & 65 \\
\hline $4 d$ & $\mathrm{Cl}$ & $2-\mathrm{CH}_{3}$ & 1.0 & $125-126$ & 85 & 69 \\
\hline $4 e$ & $\mathrm{Cl}$ & $4-\mathrm{CH}_{3}$ & 1.0 & $152-153$ & 91 & 71 \\
\hline $4 f$ & $\mathrm{Cl}$ & 4- $\mathrm{CH}_{3} \mathrm{CO}$ & 1.5 & $160-161$ & 95 & 73 \\
\hline $4 \mathrm{~g}$ & $\mathrm{Cl}$ & 3-Cl-4-F & 1.0 & $100-101$ & 87 & 68 \\
\hline 4h & $\mathrm{Cl}$ & $2,4-\mathrm{Cl}_{2}$ & 1.0 & $120-121$ & 85 & 69 \\
\hline $4 \mathbf{i}$ & $\mathrm{Cl}$ & $3-\mathrm{CH}_{3}$ & 1.0 & $122-123$ & 89 & 65 \\
\hline $4 j$ & $\mathbf{H}$ & $2-\mathrm{Cl}$ & 0.5 & $90-91$ & 92 & 72 \\
\hline $4 k$ & $\mathrm{H}$ & 4-Br & 1.0 & $109-110$ & 90 & 71 \\
\hline 41 & H & $2-\mathrm{CH}_{3}$ & 1.0 & $121-122$ & 92 & 78 \\
\hline $4 m$ & $\mathrm{H}$ & 4- $\mathrm{CH}_{3}$ & 1.0 & $101-102$ & 88 & 74 \\
\hline $4 n$ & $\mathrm{H}$ & 4- $\mathrm{CH}_{3} \mathrm{CO}$ & 1.5 & $152-153$ & 90 & 69 \\
\hline $4 p$ & $\mathbf{H}$ & $3-\mathrm{Cl}-4-\mathrm{F}$ & 1.0 & $110-111$ & 81 & 68 \\
\hline $\mathbf{4 q}$ & $\mathrm{H}$ & $2,4-\mathrm{Cl}_{2}$ & 1.0 & $102-103$ & 86 & 65 \\
\hline $4 r$ & $\mathrm{H}$ & $4-\mathrm{Cl}$ & 1.0 & $126-127$ & 84 & 69 \\
\hline
\end{tabular}




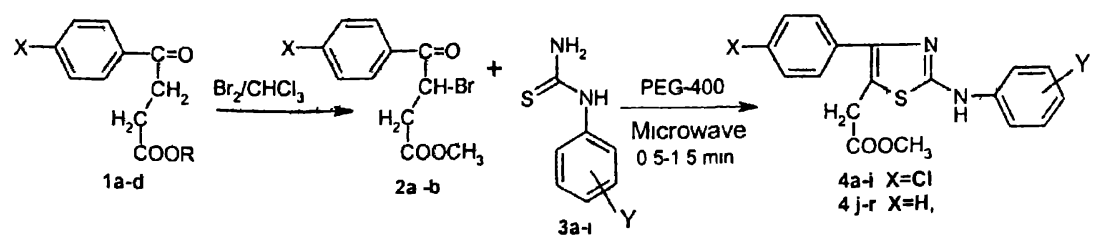

1a R = H, X = Cl; 1b R = H, X = H; 1c R $=\mathrm{CH}_{3}, \mathrm{X}=\mathrm{Cl} ; 1 \mathbf{d} \mathrm{R}=\mathrm{CH}_{3}, \mathrm{X}=\mathrm{H} ; 2 \mathrm{a} X=\mathrm{Cl} ; 2 \mathbf{b} \mathrm{X}=\mathrm{H} ; 3 \mathbf{a} \mathrm{Y}=\mathrm{H} ; 3 \mathbf{b ~ Y}=2-\mathrm{Cl}$; $3 \mathrm{c} \mathrm{Y}=4-\mathrm{Br} ; 3 \mathrm{~d} \mathrm{Y}=2-\mathrm{CH}_{3} ; 3 \mathrm{e} \mathrm{Y}=4-\mathrm{CH}_{3} ; 3 \mathrm{f} \mathrm{Y}=4-\mathrm{COCH}_{3} ; 3 \mathrm{~g} \mathrm{Y}=3-\mathrm{Cl}-4-\mathrm{F} ; 3 \mathrm{~h} \mathrm{Y}=2,4-\mathrm{Cl}_{2} ; 3 \mathrm{i} \mathrm{Y}=2-\mathrm{ClI}_{3}$

Scheme 1

Then the reaction mixture was cooled to room temperature, triturated with $10 \%$ sodium carbonate solution (50 $\mathrm{ml}$ ), allowed to stand for $15 \mathrm{~min}$ and then filtered. The solid was washed with water, dried and crystallized from ethanol to yield thiazole-5-acetate $4 \mathrm{a}(1 \mathrm{~g}, 93 \%)$ as white solid, m.p. $119-120^{\circ} \mathrm{C} ; \gamma_{\max } 3280(\mathrm{~N}-\mathrm{H}), 1720(>\mathrm{C}=\mathrm{O}$, ester), 3025, 2912 and $2875\left(\mathrm{CH}_{2}\right.$ and $\left.\mathrm{CH}_{3}\right), 1603,1586$ and $1480(\mathrm{ArC}=\mathrm{C})$ and $765 \mathrm{~cm}^{-1}(\mathrm{Ar}-\mathrm{Cl}) ; \delta 3.73(2 \mathrm{H}$, s, $\left.\mathrm{CH}_{2}\right), 3.74\left(3 \mathrm{H}, \mathrm{s}, \mathrm{CH}_{3}\right), 7.03(1 \mathrm{H}, \mathrm{t}, J 7.33 \mathrm{~Hz}$, $\left.\mathrm{ArH}_{4^{\prime}}\right), 7.13\left(2 \mathrm{H}, \mathrm{m}, \mathrm{ArH}_{3^{\prime}}\right.$ and $\left.\mathrm{ArH}_{5^{\prime}}\right), 7.24(2 \mathrm{H}, \mathrm{m}$, $\mathrm{ArH}_{2^{\prime}}$ and $\left.\mathrm{ArH}_{6^{\prime}}\right), 7.31\left(2 \mathrm{H}, \mathrm{d}, J 8.46 \mathrm{~Hz}, \mathrm{ArH}_{\mathrm{c}}\right.$ and $\left.\mathrm{ArH}_{\mathrm{e}}\right), 7.49\left(2 \mathrm{H}, \mathrm{d}, J 8.47 \mathrm{~Hz}, \mathrm{ArH}_{\mathrm{b}}\right.$ and $\left.\mathrm{ArH}_{\mathrm{f}}\right), 8.6$ $(1 \mathrm{H}, \mathrm{s}, \mathrm{NH}) ; \mathrm{MS}(\mathrm{EI}) \mathrm{m} / \mathrm{z} 358\left(\mathrm{M}^{+}\right), 360\left(\mathrm{M}^{+}+2\right)$ (Found : C, 60.30; H, 4.19; N, 7.81; S, 8.76. Calcd. for $\mathrm{C}_{18} \mathrm{H}_{5} \mathrm{O}_{2} \mathrm{~N}_{2} \mathrm{SCl}: \mathrm{C}, 60.25 ; \mathrm{H}, 4.20 ; \mathrm{N}, 7.80 ; \mathrm{S}, 8.93 \%$ ).

Other compounds $4 \mathrm{~b}-\mathrm{r}$ were synthesized in a similar manner using various phenyl thioureas.

4f ${ }^{1} \mathrm{H}$ NMR $\left(\mathrm{CDCl}_{3}\right) \delta 2.56\left(3 \mathrm{H}, \mathrm{s}, \mathrm{COCH}_{3}\right), 3.78$ $\left(3 \mathrm{H}, \mathrm{s}, \mathrm{CH}_{3}\right), 3.87\left(2 \mathrm{H}, \mathrm{s}, \mathrm{CH}_{2}\right), 7.21(2 \mathrm{H}, \mathrm{d}, J 8.69$ $\mathrm{Hz}, \mathrm{ArH}_{2^{\prime}}$ and $\left.\mathrm{ArH}_{6^{\prime}}\right), 7.34\left(2 \mathrm{H}, \mathrm{d}, J 8.24 \mathrm{~Hz}, \mathrm{ArH}_{\mathrm{c}}\right.$ and $\left.\mathrm{ArH}_{\mathrm{e}}\right), 7.53\left(2 \mathrm{H}, \mathrm{d}, J 8.26 \mathrm{~Hz}, \mathrm{ArH}_{\mathrm{b}}\right.$ and $\left.\mathrm{ArH}_{\mathrm{f}}\right), 7.83$ $\left(2 \mathrm{H}, \mathrm{d}, J 8.70-\mathrm{Hz}, \mathrm{ArH}_{3^{\prime}}\right.$ and $\left.\mathrm{ArH}_{5^{\prime}}\right), 7.95(1 \mathrm{H}, \mathrm{br}, \mathrm{NH})$; 4h $\delta 3.76\left(3 \mathrm{H}, \mathrm{s}, \mathrm{CH}_{3}\right), 3.79\left(2 \mathrm{H}, \mathrm{s}, \mathrm{CH}_{2}\right), 7.24(1 \mathrm{H}$, dd, $J 2.3$ and $\left.8.87 \mathrm{~Hz}, \mathrm{ArH}_{5^{\prime}}\right), 7.38\left(1 \mathrm{H}, \mathrm{s}, \mathrm{ArH}_{3^{\prime}}\right), 7.40$ $\left(2 \mathrm{H}, \mathrm{d}, J 8.51 \mathrm{~Hz}, \mathrm{ArH}_{\mathrm{c}}\right.$ and $\left.\mathrm{ArH}_{\mathrm{e}}\right), 7.54(2 \mathrm{H}, \mathrm{d}, J 8.49$ $\mathrm{Hz}, \mathrm{ArH}_{\mathrm{b}}$ and $\left.\mathrm{ArH}_{\mathrm{f}}\right), 8.15-8.24\left(2 \mathrm{H}, \mathrm{m}, \mathrm{ArH}_{6^{\prime}}\right.$ and $\left.\mathrm{NH}\right)$; MS : $426\left(\mathrm{M}^{+}\right), 369(100 \%), 332,297,262,196,181$, $149,137,111,102,91 ; 4 \mathbf{i} 2.24\left(3 \mathrm{H}, \mathrm{s}, \mathrm{Ar}-\mathrm{CH}_{3}\right), 3.73$ $\left(3 \mathrm{H}, \mathrm{s}, \mathrm{CH}_{3}\right), 3.77\left(2 \mathrm{H}, \mathrm{s}, \mathrm{CH}_{2}\right), 6.79\left(1 \mathrm{H}, \mathrm{s}, \mathrm{ArH}_{2^{\prime}}\right)$, $6.85(1 \mathrm{H}, \mathrm{d}, J 7.56 \mathrm{~Hz}, \mathrm{ArH}), 6.99(1 \mathrm{H}, \mathrm{m}, \mathrm{ArH}), 7.13$ $\left(1 \mathrm{H}, \mathrm{t}, J 7.70 \mathrm{~Hz}, \mathrm{ArH}_{5^{\prime}}\right), 7.30\left(2 \mathrm{H}, \mathrm{d}, J 8.52 \mathrm{~Hz}, \mathrm{ArH}_{\mathrm{c}}\right.$ and $\left.\mathrm{ArH}_{\mathrm{e}}\right), 7.51\left(2 \mathrm{H}, \mathrm{d}, J 8.48 \mathrm{~Hz}, \mathrm{ArH}_{\mathrm{b}}\right.$ and $\left.\mathrm{ArH}_{\mathrm{f}}\right)$, $8.75(1 \mathrm{H}, \mathrm{br}, \mathrm{NH})$; MS $m / z 374\left(\mathrm{M}^{+}+2\right), 372\left(\mathrm{M}^{+}\right)$, $315 / 313,278,196,181$ (100\%), 149, 137, 111, 102, 91; $4 \mathrm{j} \delta 3.76\left(3 \mathrm{H}, \mathrm{s}, \mathrm{CH}_{3}\right), 3.7\left(2 \mathrm{H}, \mathrm{s}, \mathrm{CH}_{2}\right), 6.95(1 \mathrm{H}, \mathrm{t}, J$. $7.52 \mathrm{~Hz}, \mathrm{ArH}), 7.2-7.4(5 \mathrm{H}, \mathrm{m}, \mathrm{ArH}), 7.65(2 \mathrm{H}, \mathrm{d}, J$ $8.15 \mathrm{~Hz}, \mathrm{ArH}), 8.1(1 \mathrm{H}, \mathrm{d}, J 8.20 \mathrm{~Hz}, \mathrm{ArH}) ; 4 \mathrm{k} \delta 3.73$ $\left(3 \mathrm{H}, \mathrm{s}, \mathrm{CH}_{3}\right), 3.75\left(2 \mathrm{H}, \mathrm{s}, \mathrm{CH}_{2}\right), 7.16-7.24(5 \mathrm{H}, \mathrm{m}$, ArH), $7.35\left(2 \mathrm{H}, \mathrm{d}, J 8.45 \mathrm{~Hz}, \mathrm{ArH}_{3^{\prime}}\right.$ and $\left.\mathrm{ArH}_{5^{\prime}}\right), 7.51$ $\left(2 \mathrm{H}, \mathrm{d}, J 8.43 \mathrm{~Hz}, \mathrm{ArH}_{2^{\prime}}\right.$ and $\left.\mathrm{ArH}_{6^{\prime}}\right), 7.94(1 \mathrm{H}, \mathrm{br}, \mathrm{NH})$; MS $m / z 404\left(\mathrm{M}^{+}+2\right), 402\left(\mathrm{M}^{+}\right), 345 / 343(100 \%), 331 /$ $329,264,162,147,115,103,77 ; 4$ p $\delta 3.75$ (3II, s, $\left.\mathrm{CH}_{3}\right), 3.76\left(2 \mathrm{H}, \mathrm{s}, \mathrm{CH}_{2}\right), 6.80-6.90(2 \mathrm{H}, \mathrm{m}, \mathrm{ArH}), 7.0$ (1H, m, Hz, ArH), 7.28-7.31 (3H, m, ArH), 7.50-7.54 (2H, m, ArH), 9.4-9.7 (1H, br, NH); 4q $\delta 3.76(3 \mathrm{H} . \mathrm{s}$, $\left.\mathrm{CH}_{3}\right), 3.83\left(2 \mathrm{H}, \mathrm{s}, \mathrm{CH}_{2}\right), 7.23(1 \mathrm{H}$, dd, $J 2.36$ and 8.86 $\left.\mathrm{Hz}, \mathrm{ArH}_{5^{\prime}}\right), 7.35-7.39(2 \mathrm{H}, \mathrm{m}, \mathrm{ArH}$ and $\mathrm{NH})$. 7.41$7.46(2 \mathrm{H}, \mathrm{m}, \mathrm{ArH}), 7.58-7.61(3 \mathrm{H}, \mathrm{m}, \mathrm{ArH}), 8.18(1 \mathrm{H}$, $\mathrm{d}, J 8.87 \mathrm{~Hz}, \mathrm{ArH}_{6^{\prime}}$ ).

\section{Acknowledgement}

The authors are thankful to Prof. B. G. Shivananda, Principal, Al-Ameen College of Pharmacy, Bangalore for providing facilities and to Dr. G. Bagavant for his support.

\section{References}

1. V. Paola, G. Athina, I. Matteo, B. Bernadetta, P. Graziella, C. A. Cabras and P. L. Colla, Bioorg. Med. Chem., 2003, 11, 4785; B. S. Holla, K. V. Malini, B. S. Rao, B. K. Sarojini and N. S. Kumari, Eur. J. Med. Chem., 2003, 38, 313.

2. B. Tjeed, J. Vallugarda and R. Emound, J. Med. Chem. 2002, 45, 3813.

3. K. M. Woods, C. K. Wade, K. I. Hulkower and R. L. Bel! Bioorg. Med. Lett., 2001, 111, 1325; K. Balakrishna, M. R Abdul and B. David, Archi. Der. Pharmazie, 2001, 334, 263; A. R. Maarouf, M. K. Qoot, N. Boulatara and A. R. ElNaddaf, Alexandria J. Pharm. Sci., 2001. 15, 77: N. Ulusoy; G. Capon, G. Outk and M. Kiraz, Boll. Cim. Farm., 2001, 39, 167.

4. F. G. Fabiola, D. Lakshminarasiman, P. Vasantha and N. Kuppaswamy, Current Science, 2000, 80, 26.

5. N. Lozach, "Forty Years of Heterocyclic Sulphur Chemistry" in 'Sulphur Reports', 1990, $10,7$.

6. J. Metzger, "In Comprehensive Heterocyclic Chemistry-IV", eds. Kevin and T. Potts, Pergmon Press, New York, 1984. Vol. 6, 294.

7. R. Gedye, F. Smith, K. Westway, H. Ali, I. Baldidera, L. Laberge and J. Rousell, Tetrahedron Lett., 1986, 27. 279. 
8. A. Hoz, A. D. Ortis, A. Moreno and F. Langa, Eur. J. Org. Chem., 2000, 22, 3659; S. V. Rajendra, Tetrahedron, 2002, 58, 1235; P. Laurence and L. Andre, Tetrahedron, 2001, 57. 9199; L. Pelle, T. Jason, W. Bernard and W. Jacab, Tetrahedron, 2001, 57, 9225.

9. A. K. Bose, M. S. Manhas, M. Ghosh, V. S. R. Shah, S. S. K Tabey and Z. U. Lipkkowaska, Heterocycles, 1990, 30, 741; B. K. Banik, A. K. Bose, M. S. Manhas and E. W. Robb, Heterocycles, 1997, 44, 405; B. K. Banik, A. K. Bose, N. Lavliskaia, M. Jayaraman and M. S. Manhas, Chem. Tech.,
1997, $27,18$.

10. S. Antonia, L. Sonia, A. Francesca, A. Enrico. G. Marina and D. Michele, Eur. J. Med. Chem., 1999, 34, 1003.

11. B. K. Banik, K. J. Barakat, D. R. Wagle, M. S. Manhas and A. K. Bose, J. Org. Chem., 1999, 64, 5746.

12. W. E. Truce and L. B. Lindy, J. Org. Chem., 1961, 26, 1463 . 